\title{
CFD SIMULATIONS OF TILTROTOR CONFIGURATIONS IN HOVER
}

\author{
Mark A. Potsdam \\ Roger C. Strawn \\ U.S. Army Aeroflightdynamics Directorate (AMCOM) \\ Moffett Field, CA \\ potsdam@nas.nasa.gov \\ rstrawn@mail.arc.nasa.gov
}

\begin{abstract}
Navier-Stokes computational fluid dynamics calculations are presented for isolated, half-span, and full-span V-22 tiltrotor hover configurations. These computational results extend the validity of CFD hover methodology beyond conventional rotorcraft applications to tiltrotor configurations. Computed steady-state, isolated rotor performance agrees well with experimental measurements, showing little sensitivity to grid resolution. However, blade-vortex interaction flowfield details are sensitive to numerical dissipation and are more difficult to model accurately. Time-dependent, dynamic, half- and full-span installed configurations show sensitivities in perfornance to the tiltrotor fountain flow. As such, the full-span configuration exhibits higher rotor perfornance and lower airframe downioad than the half-span configuration. Half-span rotor installation trends match available half-span data, and airframe downloads are reasonably well predicted. Overall, the CFD solutions provide a wealth of flowfield details that can be used to analyze and improve tiltrotor aerodynamic performance.
\end{abstract}

\section{NOTATION}

a

A

speed of sound

rotor disk area, $\pi \mathrm{R}^{2}$

local chord length

$\mathrm{C}_{\mathrm{Q}}$ rotor torque coefficient, $\mathrm{Q} / \rho(\Omega \mathrm{R})^{2} \mathrm{RA}$

$\mathrm{C}_{\mathrm{T}} \quad$ rotor thrust coefficient, $\mathrm{T} / \rho(\Omega \mathrm{R})^{2} \mathrm{~A}$

$\mathrm{DL} / \mathrm{T}$ airframe download divided by total thrust

FM rotor figure of merit, $\mathrm{C}_{\mathrm{T}} \sqrt{\mathrm{C}_{\mathrm{T}} / 2} / \mathrm{C}_{\mathrm{Q}}$

M Mach number, v/a

$\mathrm{M}^{2} \mathrm{c}_{\mathrm{n}} \quad$ blade section nomal force coefficient times Mach number squared, $\mathrm{N} / \frac{1}{2} \rho \mathrm{a}^{2} \mathrm{c}$

$N$ blade section normal force

Q rotor torque

$r$ radial coordinate

$\mathrm{R} \quad$ blade radius

Re Reynolds number at the rotor tip, $p(\Omega R) c_{\text {tip }} / \mu$

T rotor thrust

$v \quad$ local velocity

$x \quad$ streamwise coordinate ( $+a f t)$

y spanwise coordinate (+right)

$z \quad$ normal coordinate (+up)

r circulation

$\Theta \quad$ blade collective angle at $r / R=0.75$, degrees

$\rho \quad$ air density

$\sigma \quad$ rotor solidity, $N_{b} c_{\text {ref }} / \pi R$

Presented at the American Helicopter Society $58^{\text {th }}$ Annual Forum, Montreal, Canada, June 11-13, 2002. Copyright $\odot 2002$ by the American Helicopter Society International, Inc. All rights reserved.

\author{
$\Psi \quad$ azimuthal angle, degrees \\ $\omega$ vorticity, $1 / \mathrm{sec}$ \\ $\Omega \quad$ rotor rotational speed, $\mathrm{rad} / \mathrm{sec}$
}

\section{INTRODUCTION}

Tiltrotor aircraft are recognized for their ability to significantly change both the military and civilian aviation transportation landscapes. The range and speed of a turboprop airplane is augmented by the ability to operate in and out of confined areas like a helicopter. For civilian operations this means reduced impact on an already overloaded airspace system and reduced infrastructure costs. For military operations, increased payload and range with reduced aerial refueling operations are possible when compared with helicopters currently performing the same remote area missions. The V-22 Osprey is the first production military tiltrotor aircraft.

As with most new aircraft configurations, analysis tools need to be validated for regions beyond their conventional operation. In hover, tiltrotors differ significantly from helicopter rotors, which operate solely in edgewise flight, due to their highly twisted, low aspect ratio blades and high disc loading. These design aspects arise because the blades must operate in both propeller and helicopter rotor mode. Thick inboard airfoil sections display stall delay due to three-dimensional and centrifugal effects. In addition, airfame download prediction is more critical for tiltrotors than for conventional rotorcraft. The ability to accurately analyze and understand these features is a critical requirement for optimum tiltrotor design. For example, a 0.01 change in figure of merit (FM), a measure of hover 
efficiency, is equivalent to approximately $380 \mathrm{lbs}$. in useful load at constant power for a twin engine $V-22\left(C_{T}=0.014\right)$. In addition, about $10 \%$ of the rotor thrust is lost to airfame download for tiltrotor configurations.

This study aims to investigate high-fidelity computational fluid dynamics (CFD) methods for predicting the performance of tiltrotors in hover, both for isolated rotors and full configuration aircraft. CFD methodologies have been investigated and applied extensively to conventional helicopter rotors. In this paper, they will be applied to two model rotor experimental test cases and to a full aircraft V-22 configuration. The aim is to extend the validity of the analysis methods, enabling investigation of current full-scale tiltrotor aircraft, exploration of new design concepts, and development and better understanding of wind tunnel tests.

Previous computational work on helicopter hover is extensive. Recent works in the field include Wake and Baeder [1], Beaumier, Pahlke, and Celli [2], and Pomin and Wagner [3]. Using the Navier-Stokes overset grid methodologies employed here, Strawn [4] showed good performance prediction for a 4-bladed UH-60 rotor. However, even with 64 million grid points for a quarter domain, the detailed wake geometry was not captured correctly, and grid convergence was only shown for integrated quantities.

Previous work on tiltrotor configurations in hover is more limited, in part, due to a smaller number of tiltrotor configurations and experimental databases. An early NavierStokes simulation using a single block grid $(240,000$ grid points), momentum-source actuator disk, and a simplified half-span wing geometry [5] produced reasonable rotor performance, but download was significantly overpredicted. Numerous tiltrotor hover computations have been performed by Rajagopalan [6,7]. In these works, non-body-fitted Cartesian grids and time-averaged, momentum-source modeling of the rotor disk are used in efficient NavierStokes analyses of isolated (400,000 grid points) and installed $(700,000$ grid points) tiltrotor configurations. Isolated rotor performance is accurately predicted except at high thrust conditions, as are the incremental installation effects of half-span models. Meakin [8] computed the flow for a V-22 rotor in the presence of a half-span wing using the Navier-Stokes moving body, overset grid (2.5 million grid points) methodology employed here. Download was accurately predicted, but figure of merit was underestimated, although the comparison was made for slightly different rotors.

Results in this paper will show validation of CFD for isolated tiltrotor hover performance by comparison with experimental test data. Effects of flow solver operation and grids will be investigated in detail to determine appropriate operational guidelines. Details of the three-dimensional isolated hover flowfield will also be studied - data not available from lower fidelity comprehensive codes or momentum-source models. The CFD methodology will then be applied to investigate the hover aerodynamics of half- and full-span tiltrotor aircraft configurations, including installed rotor performance, airframe download, and detailed flow physics.

\section{METHODOLOGY}

CFD calculations use the Reynolds-averaged NavierStokes computational fluid dynamics code OVERFLOW-D [9]. It is based on the OVERFLOW code, which was developed at NASA and has been applied to a wide range of fluid dynamics problems. OVERFLOW-D includes major modifications for rigid body motion of components. Solutions are computed on structured, overset grids using body-conforming "near-body" grids and automatically generated Cartesian "off-body" grids [10] in the wake and farfield. Several modifications to the code for calculations of hovering rotors have been made [11]. In particular, isolated rotor flows can be computed as steady-state problems in a blade-fixed reference frame with the addition of a rotational source term to the finite difference equations.

More complex hover configurations require the timeaccurate modeling of the moving rotor blades. User-defined subroutines prescribe the arbitrary six degree-of-freedom blade motion. Grid motion requires recalculation of the domain connectivity, including hole cuts and interpolation coefficients, at each time step, as the near-body grids move through the stationary off-body grids. Reuse of information from the previous time step enables order of magnitude speed-ups compared to domain connectivity solutions from scratch. Using this technique, the domain connectivity work can be efficiently performed in less than $20 \%$ of the time required for the flow solver.

Solutions are computed on large parallel computers or a network of PCs/workstations communicating with the Message Passing Interface (MPI) protocol. Both the domain connectivity and flow solver modules have been parallelized for efficient, scalable computations using MPI [12]. Coarse grain parallelization on large numbers of processors is achieved by distributing grids among the processors, and, if necessary, splitting them as appropriate into smaller blocks to prevent bottlenecks. Boundaries that are created in the splitting process have explicit boundary conditions, similar to intergrid boundaries of the original grid system.

\section{ISOLATED ROTOR MODELING}

\section{Isolated Rotor Test Cases}

A first step in the application of CFD for tiltrotor configurations in hover is the investigation of isolated tiltrotor Navier-Stokes CFD analysis capabilities. 
Computational simulations were run for two experimentally tested isolated rotors, TRAM and JVX.

The Tilt Rotor Aeroacoustics Model (TRAM) is an extensive wind tunnel model constructed to facilitate future tiltrotor aeromechanics research. The geometry is a 0.25-scale V-22 right-hand nacelle and 3-bladed rotor with geometric and dynamic scaling. The isolated TRAM rotor was tested in the Duits-Nederlandse Windtunnel Large Low-speed Facility (DNW-LLF) in the spring of 1998. It provided a significant new source of aeroacoustics, performance, and structural loads data for validation of tiltrotor analyses. Among the aerodynamics data acquired were rotor performance and blade pressures. Hover runs were performed at nominal tip Mach numbers of 0.58 and 0.62 . Rotor operational limitations resulted in the 0.62 hover data rather than the V-22 hover tip Mach number of 0.72 . Hover testing was performed with the rotor shaft axis at 0 degrees (helicopter mode) and -76 degrees (airplane mode). Support blockage and interference are reduced in airplane mode. Performance data in this mode are considered to be more accurate based on discussions by Johnson [13] and are used for comparisons with the computational results. Details of the test and data reduction are described in References 14 and 15 .

The JVX is a 0.658 -scale model of an early V-22 configuration with higher solidity obtained by increasing the chord $8.4 \%$ along the span. The isolated rotor was tested in the NASA Ames Research Center Outdoor Aerodynamic Research Facility (OARF) in 1984 [16]. Good data repeatability and reduced interference effects of the outdoor testing apparatus make this a high-quality dataset for computational validation. Data were obtained closer to the actual V-22 hover tip Mach number at 0.68 and larger tip Reynolds number of 5.9 million.

The main physical characteristics of the two rotors are presented in Table 1, and nominal test parameters in hover are summarized in Table 2. Both rotors use $\mathrm{V}-22$ airfoil sections and twist and thickness distributions along most of the span $(r / R>0.25)$. Blade root fairings were slightly modified for each rotor. The large amount of nonlinear twist is typical of tiltrotors but significantly different from helicopter configurations. Details of the models and geometries have been described in Reference [13]

\section{Isolated Hover Analysis}

Rotor performance comparisons were made for both test cases. In addition, the TRAM rotor was used to investigate, in detail, the effects of grid topology, grid resolution, and flow solver algorithms as well as flowfield details.

The 3-bladed TRAM rotor is shown in Figure 1. The inboard upper surface cuff geometry $(r / R<0.27)$ was obtained from a digitized scan of one of the model blades,
Table 1. TRAM and JVX rotor physical characteristics

\begin{tabular}{lll}
\hline \hline & \multicolumn{1}{c}{ TRAM } & \multicolumn{1}{c}{ JVX } \\
\hline description & $\begin{array}{l}0.25 \text {-scale V-22 } \\
\text { nacelle and rotor }\end{array}$ & $\begin{array}{l}0.658 \text {-scale V-22 } \\
\text { development rotor }\end{array}$ \\
\hline rotor radius & 57 in. & 150.0 in. \\
\hline solidity, $\sigma$ & 0.105 & 0.1138 \\
\hline tip chord, $\mathrm{c}_{\text {tip }}$ & $5.5 \mathrm{in}$. & 15.79 in. \\
\hline airfoil & & $\mathrm{V}-22 \mathrm{XN}$ series \\
sections & modified root & $\begin{array}{l}8.4 \% \text { chord } \\
\text { increase }\end{array}$ \\
& fairing & 32 to -6 degrees, nonlinear \\
\hline twist &
\end{tabular}

Table 2. TRAM DNW and JVX OARF nominal test parameters in hover.

\begin{tabular}{|c|c|c|}
\hline & TRAM & JVX \\
\hline tip Mach number & $0.58,0.62$ & 0.68 \\
\hline tip $\operatorname{Re}$ (million) & 2.1 & 5.9 \\
\hline $\mathrm{C}_{\mathrm{T}} / \sigma$ range & $0.05-0.17$ & $0.0-0.16$ \\
\hline collective range & $3-17$ deg & $-4-17$ deg \\
\hline
\end{tabular}

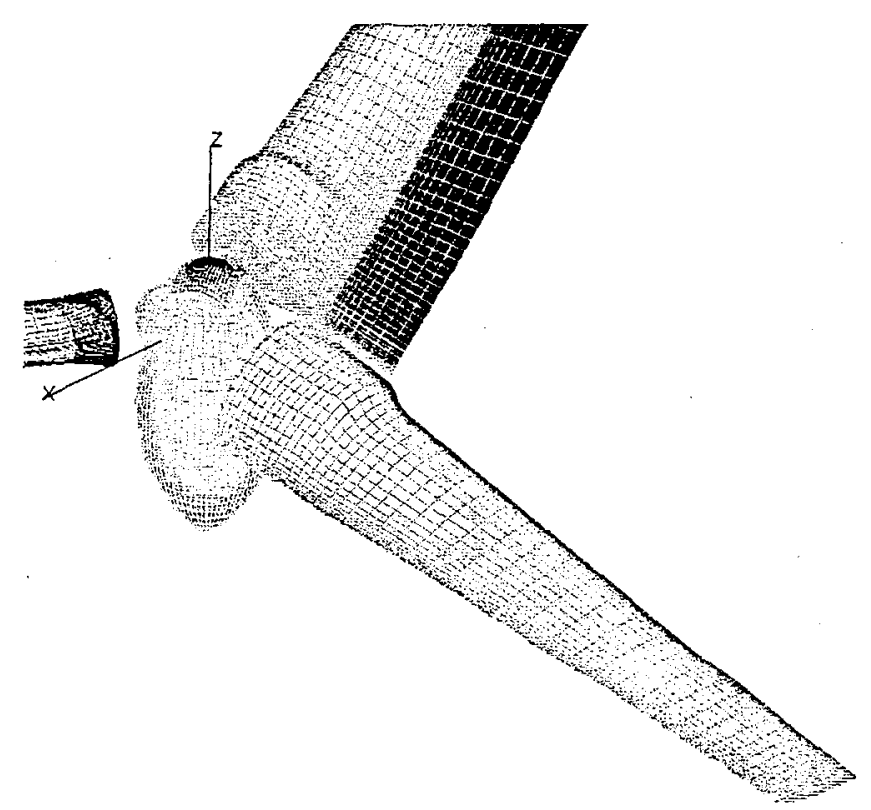

Figure 1. Isolated TRAM geometry and baseline surface grids (every other point).

while the remainder of the model used the CAD definition. The IVX is defined by CAD data. Elastic blade effects are minor in hover [13] and are not modeled. The centerbody is a V-22 spinner with a faired boattail. The hub is modeled without connecting flexbeam. The effect of this geometry simplification on the overall solution should be minimal due to low velocities in this region. The blade extends from $0.10 \mathrm{R}$ to $1.0 \mathrm{R}$ and is physically separated from the spinner by a distance of $0.026 R$. 
Table 3. Grid Parameters.

\begin{tabular}{|c|c|c|}
\hline & Baseline & Alternative \\
\hline blade topology & $\begin{array}{l}\text { C-mesh: } \\
181 \times 94 \times 65^{2} \text { blade } \\
111 \times 67 \times 65 \text { hub } \\
109 \times 47 \times 65 \text { tip }\end{array}$ & $\begin{array}{l}\text { O-mesh: } \\
121 \times 94 \times 65 \text { blade } \\
81 \times 67 \times 65 \text { hub } \\
88 \times 49 \times 65 \text { tip }\end{array}$ \\
\hline $\begin{array}{l}\text { near-body } \\
\text { spacing }\end{array}$ & $\begin{array}{l}\text { chordwise: } \\
.004-.0012 \mathrm{c}-\mathrm{LE} \\
0.0008 \mathrm{c}-\mathrm{TE} \\
\text { normal: } 0.0002 \\
\text { spanwise: } \\
0.004 \mathrm{R}-\text { root } \\
0.0024 \mathrm{R}-\mathrm{tip} \\
0.019 \mathrm{R}-\mathrm{max}\end{array}$ & $\begin{array}{l}2 / 3 \text { of baseline: } \\
271 \times 139 \times 97 \text { blade } \\
166 \times 101 \times 97 \text { hub } \\
163 \times 71 \times 97 \text { tip }\end{array}$ \\
\hline $\begin{array}{l}\text { near-body outer } \\
\text { boundary }\end{array}$ & \multicolumn{2}{|c|}{$\sim c_{\text {tip }}$} \\
\hline $\begin{array}{l}\text { off-body } \\
\text { spacing }\end{array}$ & $0.10 c_{\text {tip }}$ & $0.05,0.14 c_{\text {tip }}$ \\
\hline outer boundary & \multicolumn{2}{|c|}{$5 \mathrm{R}$} \\
\hline
\end{tabular}

${ }^{a}$ chordwise $\mathrm{x}$ spanwise $\mathrm{x}$ normal

\section{Baseline Grid Generation}

The baseline grid systems for the TRAM and JVX rotors are similar, so only details of the TRAM configuration will be provided. In the Chimera methodology, overset, structured near-body grids are generated about the geometry. They extend approximately one tip chord $\left(c_{\text {tip }}\right)$ away from the body and include sufficient resolution to capture boundary layer viscous effects. The baseline surface grids are also shown in Figure 1. They use C-mesh topology blades and tip caps. Grid spacing parameters in all directions are detailed in Table 3. The first four points away from the blade surfaces have a constant spacing, verified to produce a $y^{t} \leq 1$. These spacing parameters closely match those determined for accurate drag prediction on transonic fixed wings using OVERFLOW [17].

Off-body Cartesian grid generation is automatically performed by OVERFLOW-D. The finest off-body spacing for the baseline grid is $0.10 c_{\text {tip. }}$. This level-1 grid surrounds the blades and extends $\pm 1.23 \mathrm{R}$ in $\mathrm{x}$ and $\mathrm{y}$ and $-0.58,+0.23$ in $z$. It is manually specified in order to contain the entire wake. A total of five progressively coarser levels are generated out to the farfield boundary, which is placed at $5 \mathrm{R}$ in all directions from the center of the domain. The grid spacings differ by a factor of two between each mesh level. The baseline grid contains 15.9 million (M) points: $6.2 \mathrm{M}$ near-body and 9.7M off-body. Where grid points fall inside the geometry, hole cutting is employed to blank out these points. A cut through the grid system in Figure 2 shows the near- and off-body grids, hole cuts, and overlap.

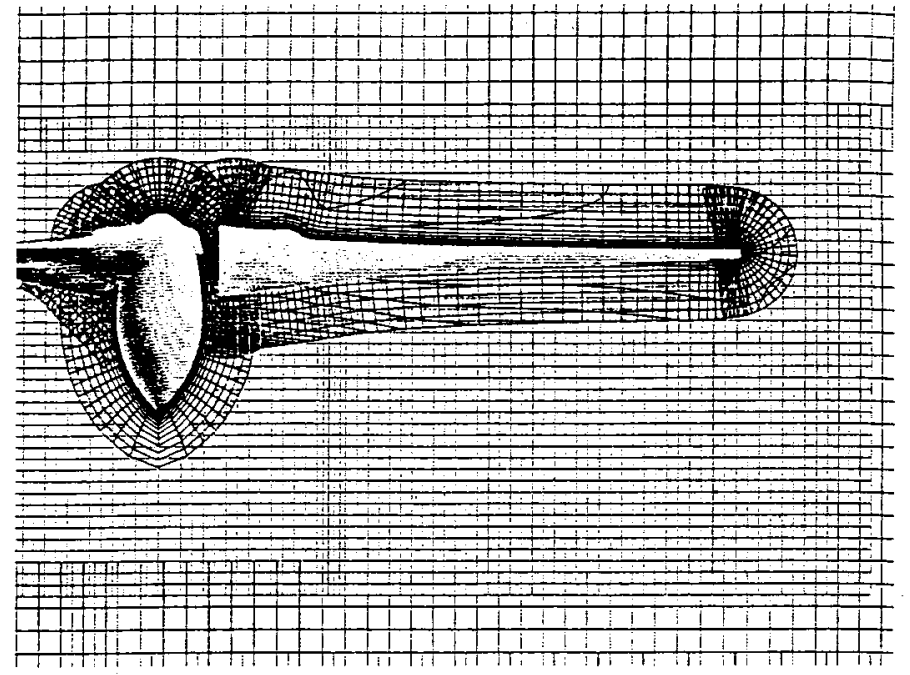

Figure 2. Slice through isolated TRAM volume grids (every other point). black - near-body, red - finest level off-body, blue - coarser level off-body

\section{Parametric Grid Variation}

In order to determine the sensitivity of calculated tiltrotor performance to grid effects, several alternative grids were generated for the TRAM configuration. Total numbers of points range from 11.1 to 37.4 million. For the blades, an $\mathrm{O}$-mesh topology was generated with spacings similar to the $\mathrm{C}$-mesh. If not for the $\mathrm{O}$-mesh topology requirement to place five points on the blunt trailing edge, the C-mesh chordwise trailing edge spacings could have been relaxed slightly. Because of the reduced wake resolution, the O-mesh topology blades and caps have $26 \%$ fewer points. Additionally, O-meshes are significantly easier to generate so that their accuracy is worth investigating.

Grid density was varied in both the near-body (NB) and off-body (OB) meshes. Coarser off-body grids with $0.14 c_{\text {tip }}$ spacing in the finest level and correspondingly coarser spacings in the farfield were generated. A finer offbody grid with $0.05 c_{\text {tip }}$ spacing was manually generated inside the finest off-body grid level of the baseline grid. It was generated in cylindrical rather than Cartesian coordinates with extent $1.12 \mathrm{R}$ in $\mathrm{r}$ and $-0.19 \mathrm{R}$, $+0.15 \mathrm{R}$ in z. Cylindrical coordinates were used so that the fine spacing would always be normal to the rotational flow: Azimuthal spacing is 0.37 degrees $\left(0.067 c_{\text {tip }}\right.$ at the blade tip). Finally, near-body blades and caps were generated with 1.5 times the number of points in all directions (2/3 of the baseline spacings) and combined with the fine cylindrical off-body grid. The different grid systems investigated are detailed in Table 4. 
Table 4. Grid systems.

\begin{tabular}{lcccc}
\hline \hline grid & $\begin{array}{c}\text { near- } \\
\text { body } \\
\text { spacing }\end{array}$ & $\begin{array}{c}\text { finest } \\
\text { off-body } \\
\text { spacing }\end{array}$ & $\begin{array}{c}\text { off-body } \\
\text { points } \\
\%\end{array}$ & $\begin{array}{c}\text { \# points } \\
\text { (million) }\end{array}$ \\
\hline C-mesh & baseline & $0.10 c_{\text {tip }}$ & 61 & 15.9 \\
\hline $\begin{array}{l}\text { coarse off-body } \\
\text { fine off-body }\end{array}$ & baseline & $0.14 c_{\text {tip }}$ & 44 & 11.1 \\
\hline $\begin{array}{l}\text { baseline } \\
\text { fine near-/off- }\end{array}$ & $\begin{array}{c}0.05 c_{\text {tip }} \\
\text { cylindrical }\end{array}$ & 74 & 24.3 \\
body & $\begin{array}{c}2 / 3 \\
\text { baseline }\end{array}$ & $\begin{array}{c}0.05 c_{\text {tip }} \\
\text { cylindrical }\end{array}$ & 47 & 37.4 \\
\hline O-mesh & baseline & $0.10 c_{\text {tip }}$ & 68 & 14.2 \\
\hline \hline
\end{tabular}

\section{Flow Solver Details}

For spatial discretization, the baseline operation of OVERFLOW-D used $4^{\text {th }}$-order central difference for the inviscid terms, $2^{\text {nd }}$-order central difference for the viscous terms, $4^{\text {th }}$-order artificial dissipation, no $2^{\text {nd }}$-order artificial dissipation, and $2^{\text {nd }}$-order grid metrics. All grids overlap sufficiently to allow double fringing, where two boundary points are interpolated from adjacent overlapping grids. This allows gradient as well as solution information to be transferred more smoothly and accurately between grids. The isolated hover solutions were run using the steady-state formulation. A characteristic boundary condition was used at the outer boundary where freestream values are approximated using a source-sink distribution [11]. An LU-SGS time advancement algorithm is combined with local time stepping to speed convergence to a steady state.

The Baldwin-Barth one-equation turbulence model is used in the near-body grids with thin-layer viscous terms added only in the computational direction normal to the surface. Turning on the thin-layer viscous terms in all three computational directions in the near-body grids produced no discernable change in the solution. Except as noted, the near-body domain is assumed fully turbulent with no transition region specified. Off-body grids as well as the centerbody were modeled using the inviscid. Euler equations in order to reduce the non-physical numerical dissipation of the wake.

In addition to grid variation, flow solver parameter variations were investigated. The first involved changes in the $4^{\text {th }}$-order dissipation coefficient. Second, for comparison with more complex applications, a moving grid, unsteady hover simulation was performed. This dynamic simulation was compared with results using the steady-state formulation. The time-accurate analysis used an implicit $1^{\text {st }}$-order Pulliam-Chaussee pentadiagonal algorithm in the near-body grids and an explicit $3^{\text {rd }}$-order 3 -stage RungeKutta scheme in the off-body grids.

\section{ISOLATED HOVER RESULTS AND DISCUSSION}

\section{NX Rotor Performance}

CFD solutions for the JVX rotor were run for a number of collective angles and a tip Mach number of 0.68 using the baseline grid system. Figures 3 and 4 compare calculated and experimental thrust, torque, and FM values. Excellent agreement is seen across the thrust range. The computed figures of merit generally underpredict the experimental values by less than 0.02 at all thrust levels.

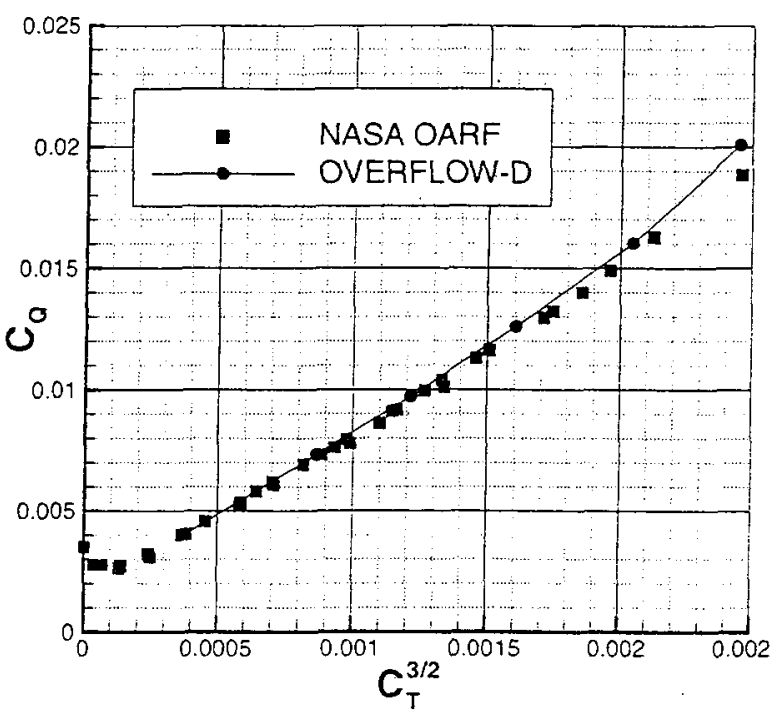

Figure 3. Isolated JVX rotor hover performance: torque vs. thrust.

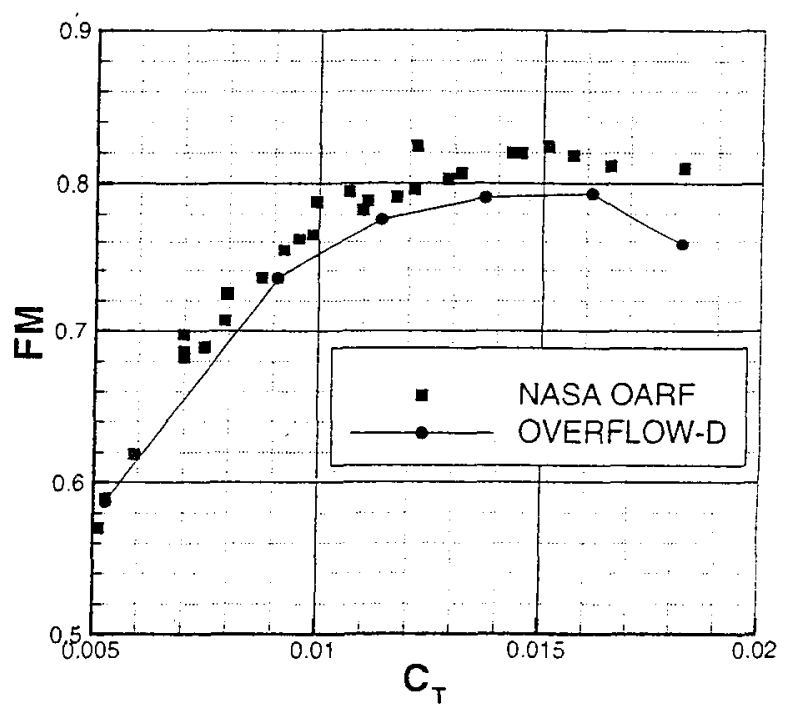

Figure 4. Isolated JVX rotor hover performance: figure of merit vs. thrust. 


\section{TRAM Rotor Performance}

CFD solutions for the TRAM rotor were run for a range of collective angles from 8 to 16 degrees with a tip Mach number of 0.625 . Comparisons of computations with the TRAM DNW hover performance data are shown in Figures 5, 6, and 7, detailing torque, FM, and collective trends with thrust. The agreement for rotor performance is again quite good, however, the slope of the $C_{T}-\Theta$ (Figure 7 ) curve is too large. There is a consistent overprediction of torque $(\sim 0.000020)$ and underprediction of figure of merit (0.01-0.02) with the correct trends predicted across the collective range.

The discrepancies are mostly due to the fully turbulent assumption. At these local chord Reynolds numbers, some laminar flow undoubtedly exists on the lower surface given the favorable pressures gradients there (see below for a discussion of blade sectional pressure distributions). Skin friction measurements on a full-scale XV-15 rotor [18] indicate that there is a possibility for laminar flow on the upper surface as well at lower collective angles. The V-22 and XV-15 rotors have similar twist and thickness distributions, albeit different planforms (tapered vs. straight) and airfoil sections. The XV-15 test tip Mach numbers of 0.56 and 0.69 equate to higher tip Reynolds numbers of 4.0-5.0 million. At collectives angles between 7 and 14 degrees, upper surface transition was seen to recede from $30 \%$ to $3 \%$ chord on average along the span of the blade.

Based on an average location of the start of adverse pressure gradients in the pressure distributions, a laminar region was specified for the 14-degree collective case. The region extends from the leading edge to $x / c=0.60$ on the lower surface and to $x / c=0.05$ on the upper surface. This transition case is plotted in Figures 5, 6, and 7 and detailed in Table 5. It produced a 0.014 increase in FM due in part to a 0.000016 decrease in the viscous component of the torque coefficient. In this case, the viscous torque contribution (.000074) is less than $5 \%$ of the total torque. As a result, accounting for boundary layer transition further improves the performance comparisons to within the range of experimental data uncertainty and should also improve the correlation at other collective angles.

\section{Blade Sectional Quantities}

Blade pressures from the TRAM test were obtained at seven radial stations during hover testing in helicopter mode and integrated to obtain section normal force distributions. Experimental and computed pressures at radial stations $r / R=0.82$ and 0.90 are compared in Figure 8. Agreement is reasonable, but at higher thrusts, upper surface pressures are consistently overpredicted. At this point, it should be noted that integration of the experimental pressures underestimates the balance thrust by

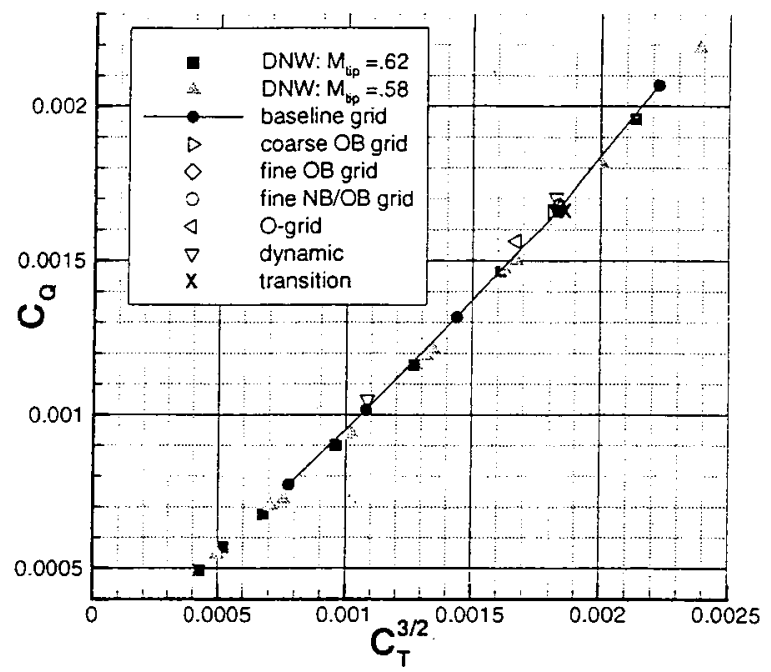

Figure 5. Isolated TRAM rotor hover performance: torque vs. thrust.

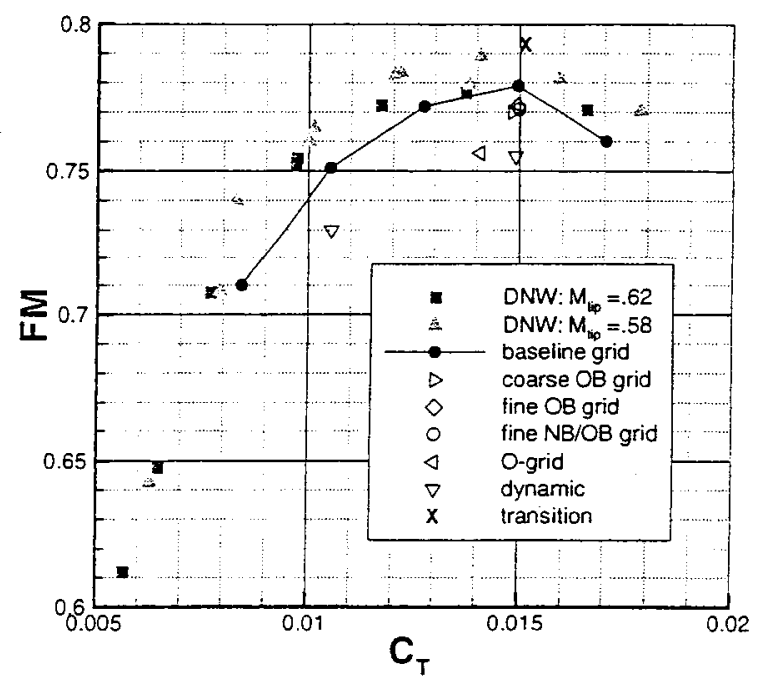

Figure 6. Isolated TRAM rotor hover performance: figure of merit vs. thrust.

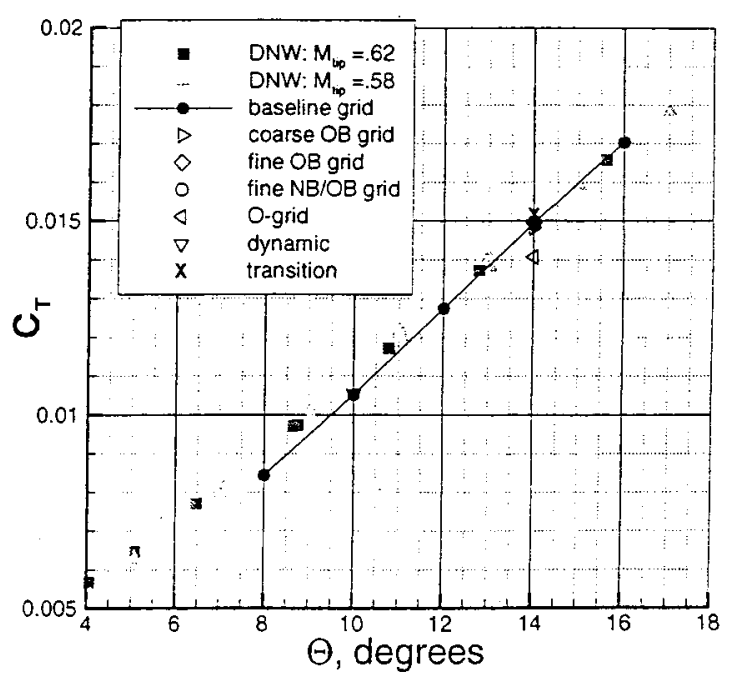

Figure 7. Isolated TRAM rotor hover performance: thrust vs. collective. 
Table 5. TRAM isolated rotor performance comparison at 14 degrees collective.

\begin{tabular}{|c|c|c|c|c|c|c|c|c|}
\hline \multirow[b]{2}{*}{ grid } & \multicolumn{3}{|c|}{ Performance } & 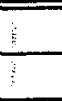 & \multicolumn{4}{|c|}{ Wake geometry (first BVI) } \\
\hline & $\mathrm{C}_{T}$ & $\mathrm{C}_{0}$ & FM & $\mathrm{r} / \mathrm{R}$ & $z / c$ & radius $/ \mathrm{c}$ & $|\omega|_{\max }$ & $\Gamma / \overline{\Omega R c}$ \\
\hline C-mesh (baseline) & .01495 & .001660 & .779 & .86 & -.50 & .85 & .017 & .49 \\
\hline transition & .01513 & .001660 & .793 & & & & & \\
\hline coarse off-body & .01483 & .001659 & .770 & .86 & -.55 & .94 & .011 & .48 \\
\hline fine off-body & .01495 & .001675 & .772 & .87 & -.53 & .55 & .037 & \\
\hline fine near-loff-body & .01498 & .001681 & .771 & .87 & -.53 & .52 & .040 & .44 \\
\hline O-mesh & .01408 & .001562 & .756 & .87 & -.53 & .82 & .017 & .45 \\
\hline dynamic & .01491 & .001705 & .755 & .86 & -.59 & .83 & .016 & .46 \\
\hline
\end{tabular}

at least $15 \%$. Errors in surface pressure data or fidelity of the surface geometry may be the cause. Experimental normal force distributions are compared with computation in Figure 9 , which serves to show that the trends with $r / R$ and collective are representative. Unfortunately, it is not clear from the pressure and normal force coefficient comparisons whether the calculated spanload distribution outboard of $r / R=0.70$ is correct. The loads on this part of the rotor are largely due to the first blade-vortex interaction (BVI). CFD calculations tend to overpredict the effects of the first BVI due to excessive dissipation of the preceding tip vortex. The influences of blade-vortex interaction and vortex dissipation on spanload are discussed below in relation to grid effects.

\section{Flowfield Details}

Detailed investigation of the TRAM CFD solutions indicates mild flow separation on the cuff and inboard part of the blade at all collective angles computed. The separated region, as determined by a contour of zero skin friction in the rotating reference frame, is shown in Figure 10 for 14 degrees collective. Surface streamlines in this reference frame are also shown and indicate three-dimensional relief is provided by the centrifugal effects forcing the flow outboard. The normal extent of the reversed flow is quite small, less than 3 percent of the local boundary layer thickness. These observations qualitatively match skin friction measurements on a full-scale XV-15 rotor at similar collective angles and $\mathrm{C}_{T} / \sigma$ [18]. Despite rotor geometric differences, separation locations at $\mathrm{r} / \mathrm{R}=0.17,0.28$, and 0.50 and the overall spanwise flow region are in generally good agreement. The cause of the separation is related to the trade-offs in tiltrotor design requiring high root twist when operating as a propeller compared with desired conventional helicopter root twist. Based on a spanwise torque distribution, there does not appear to be any performance penalty associated with the inboard separation. Further investigation of the inboard flowfield may be useful for correlating stall delay characteristics for comprehensive codes, which use modified airfoil tables, since this phenomenon is directly modeled
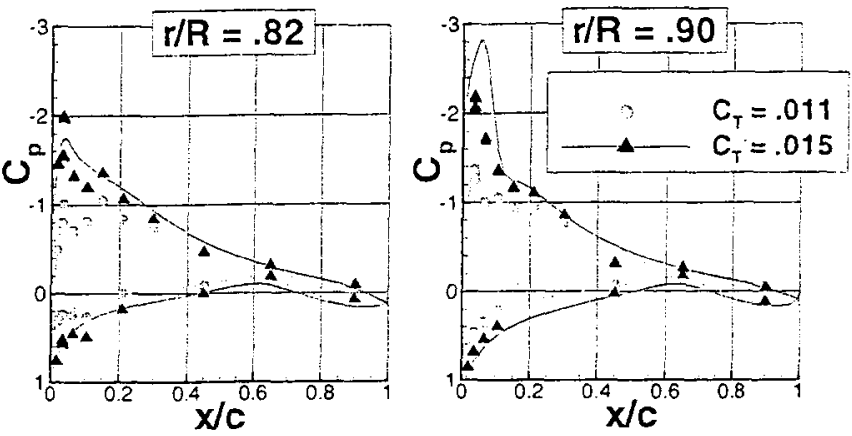

Figure 8. Isolated TRAM rotor chordwise pressure distributions. symbols - test, line - calculated

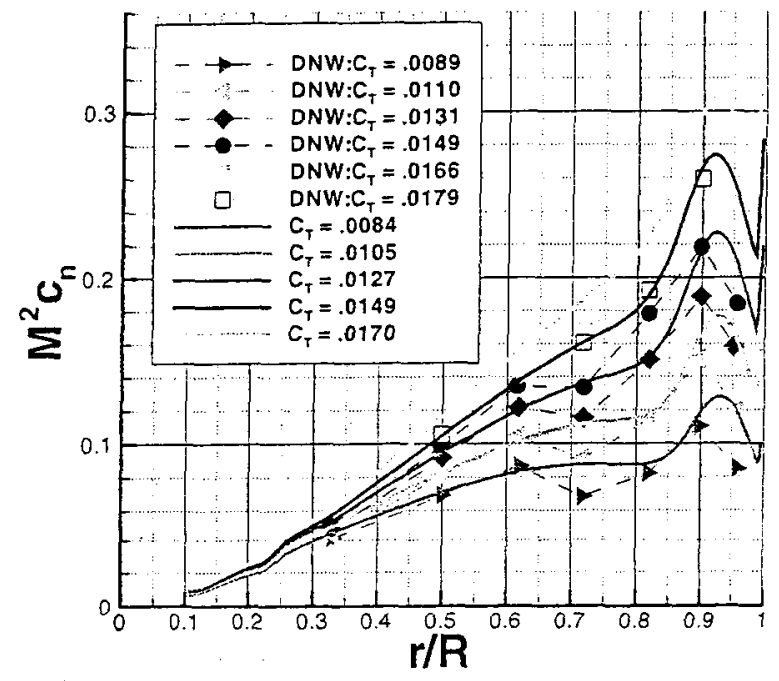

Figure 9. Isolated TRAM rotor normal force distributions. symbols - test, line - calculated

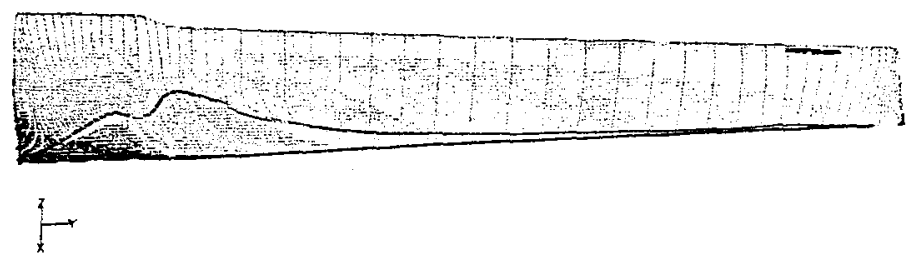

Figure 10. Isolated TRAM rotor upper surface streamlines (red) and separated region (black), $\mathrm{C}_{\mathrm{T}}=0.015$. 

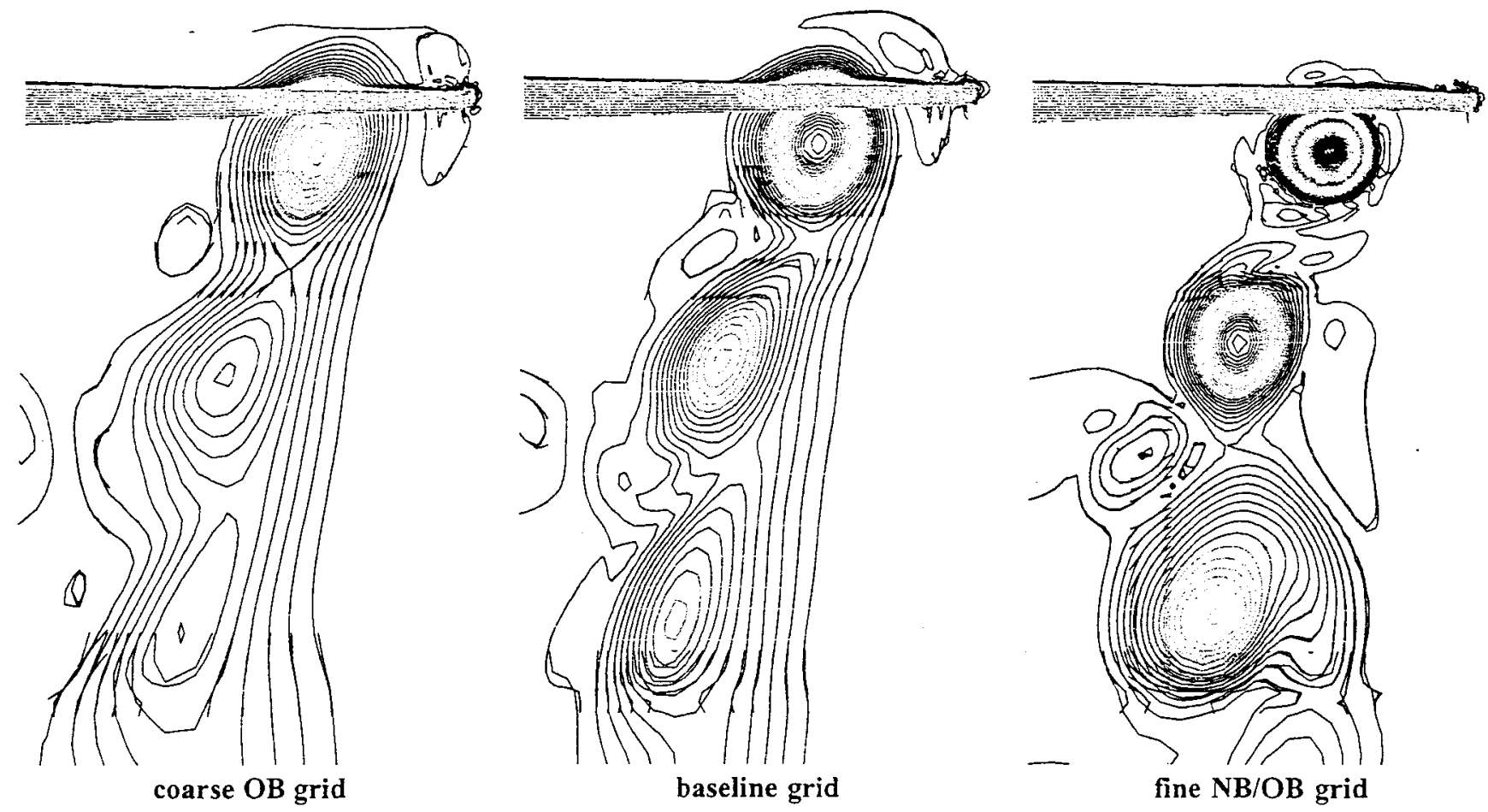

Figure 11. Isolated TRAM rotor wake vorticity at blade. blue - low, red - high

by CFD. A small separation region is also seen outboard at the foot of the shock near the leading edge.

\section{Grid Effects}

Performance results on the alternative grids are also shown in Figures 5, 6, and 7 and tabulated in Table 5 for computations at 14 degrees collective. The O-mesh underpredicts $\mathrm{C}_{\mathrm{T}}$ and $\mathrm{FM}$ compared with all the other grids. Flow visualization of the O-mesh results (not shown) indicates an immediate degradation of the wake vortex sheet due to the poor resolution behind the trailing edge. Additionally, the trailing edge details are not captured as accurately by the $\mathrm{O}$-mesh. Reduced pressure on the lower surface aft of $\mathrm{x} / \mathrm{c}=0.8$ along the entire span explains the underprediction of $C_{T}$. The spanloads and wake visualizations for the $\mathrm{O}$ - and $\mathrm{C}$-meshes are the same, indicating that the dominant problem may be the poor trailing edge treatment rather than the loss of the wake sheet from the trailing edge. Despite their ease of generation, $\mathrm{O}$-meshes are not recommended for accurate rotor blade calculations with OVERFLOW-D.

For the C-mesh grid systems, the effects of grid density on overall performance are minimal. Between the baseline and fine near-body grids, the viscous forces are the same, indicating grid convergence in the normal distributions. Differences are more apparent in the spanloads and wake structure. Table 5 also shows details of the first BVI. This first vortex encounter has a major effect on the flowfield in the outer blade region.

Figure 11 shows the wake vorticity contours for the three off-body grid densities. For the baseline grid, the first BVI vortex has a radius that is $63 \%$ larger, a peak vorticity that is $58 \%$ lower, and a miss distance that is $0.03 c_{\text {tip }}$ higher compared with the fine grid solution. The total circulation is the same for the two grid densities, indicating vorticity conservation despite the numerical dissipation. The baseline grid maintains the tip vortices until a wake age of 360 degrees, at which point they move into the next level coarser off-body mesh.

Figure 12 shows the effects of vortex dissipation on the spanload. In the baseline solution, a more diffuse and closer vortex induces higher local flow angles outboard of the BVI location $(r / R=0.86)$ and lower local flow angles inboard. The result is a peakier spanwise loading "distribution compared to the fine grid. This is supported by examination of pressure distributions. The coarse grid result shows combined effects, since the vortex is even more diffuse, significantly weaker, but has a larger miss distance. These grid convergence results agree with previous findings for helicopter rotors in hover [4]. Convergence of integrated quantities can be achieved with careful attention to grid details, however, convergence of flowfield details has not yet been realized. 


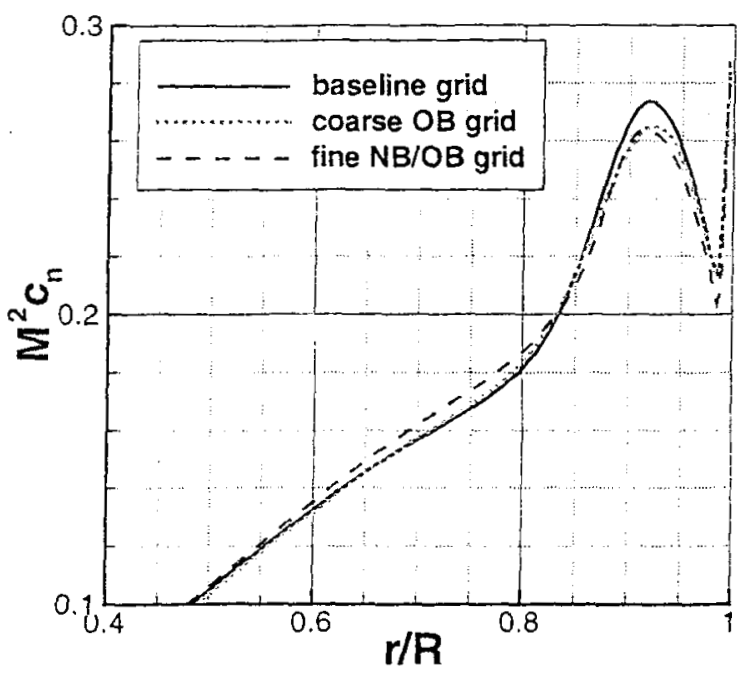

Figure 12. Isolated TRAM rotor normal force distributions for different grid densities.

\section{Flow Solver Effects}

Variation of the $4^{\text {th }}$-order dissipation coefficient showed that there is no change in the solution for values less than the baseline value used here of 0.02 . However, larger values adversely affect the performance predictions. Note that a dissipation coefficient of 0.04 is the default for OVERFLOW. Smaller values tend to make the solution more unsteady, but not unstable.

\section{Steady-State and Dynamic Formulations}

For comparison with complex, moving body problems for which there is no transformation to a steady-state reference frame, a dynamic, moving grid, hover simulation was run. Exactly the same grid system and hole cutting methodology are used. The major difference is that the nearbody blade grids move dynamically and time accurately through the stationary off-body grids. The moving gnid calculation uses 3200 iterations per revolution, 0.1125 degrees per iteration.

For complex configurations with multiple rotors, a source-sink characteristic boundary condition may not be a good model for the bottom outflow boundary. In this case, the outer boundary condition uses freestream $\left(\mathrm{M}_{\infty}=0\right)$ characteristics. Solutions using source-sink and freestream characteristic boundary conditions showed negligible differences when the outer boundaries are at least 5R away.

Performance comparisons (Table 5) show that the calculated thrust is the same between the steady and dynamic calculations, but the torque is larger for the dynamic formulation, resulting in a 0.024 reduction in FM. This is due to a difference in the miss distance of the first BVI and corresponding effects on the outboard blade loading.
In theory, there should be no difference in the solutions based on the choice of reference frame for the problem. In practice, however, several subtle differences in the methodology can arise. First, the time advancement scheme adds additional dissipation and dispersion. A time step convergence study was performed, and $\Delta \Psi=0.1125 \mathrm{deg}$ was determined to be sufficient for temporal accuracy, in agreement with the findings of other researchers [3]. No change in performance was noted for $\Delta \Psi$ values as small as $0.0281 \mathrm{deg}$. However, due to the interaction of numerical diffusion between the temporal and spatial schemes, it may be necessary to perform a convergence study simultaneously in time and space to state this conclusively.

Changing the order of accuracy of the temporal scheme in the off-body grids from the baseline $3^{\text {rd }}$-order explicit to $1^{\text {st }}$-order implicit showed little difference in overall performance. Lagged boundary condition information also affects the temporal accuracy. Grid splitting for parallel processing introduces numerous explicit Chimera boundaries into both the near-body and off-body grids. While temporal accuracy may be a cause for the steady-state vs. time-accurate discrepancies, it was not possible to produce the required magnitude of changes with the options investigated.

Second, the steady simulation uses an analytical representation to model the rotational motion. The grid speed calculation in the steady formulation is exact while the dynamic formulation uses backward differences to compute the grid velocities. The steady simulation also uses an analytical rotational source term. This centrifugal force term should balance the right hand side residual from the unsteady formulation. Due to numerical and discretization errors, the terms may be slightly out of balance, perhaps manifesting itself in a lack of conservation of angular momentum.

At this time, the discrepancies between steady-state and dynamic hover results cannot be explained. However, they have significant repercussions for the ability to analyze complex simulations and comparison back to isolated performance based on a steady formulation. Similar discrepancies were seen elsewhere [3] and remained unresolved.

\section{Computational Convergence}

Solutions were run using 48-64 processors of an IBM SP3. For steady-state simulations and depending on the configuration and collective, convergence of thrust and torque is reached after 15,000 to 30,000 iterations, requiring approximately 20 to 40 wallclock hours. The values are oscillatory within $\pm 0.5 \%$. The magnitude of the oscillations is reduced by turning off local time-stepping after initial convergence. The dynamic simulation includes a $16 \%$ penalty due to recalculation of the domain 
connectivity every iteration. Approximately 10 rotor revolutions are required to obtain converged force/moment values, equating to 32,000 iterations. Sample convergence histories for isolated steady-state (restarted from a lower collective angle) and dynamic simulations are shown below in Figure 14.

\section{INSTALLED ROTOR APPLICATION}

Investigation of isolated, hovering tiltrotors has shown that accurate rotor performance predictions (Figures 4 and 6) can be obtained given the guidelines of grid resolution and flow solver operation. This suggests that the CFD methods can be successfully applied to simulate more complex tiltrotor configurations, and, in particular, to investigate rotor installation effects.

\section{Half-span JVX Test}

The JVX rotor was tested in the presence of a 0.658-scale half-span V-22 wing, simulated fuselage fairing, and image plane. This occurred during the same test as the isolated configuration. Because there are no tunnel-to-tunnel installation effects, the configuration increments from this test should be of especially high quality. Wing pressures were recorded at six span stations and integrated to obtain download measurements. Comparisons with the TRAM rotor are made at consistent $C_{T} / \sigma$. Details of the model, test, and results can be found in References 16 and 19. Nominal test parameters in hover are the same as isolated (Table 2).

\section{Installed Hover Analysis}

A CFD model has been constructed of an installed TRAM rotor on a V-22 airframe configuration in hover. The blade geometry is the same used for the isolated hover analysis. The aircraft fuselage, wing, nacelles, and tail are actual V-22 geometry. There are some differences between the JVX half-span model described above and the full-span $\mathrm{V}-22$ as modeled here. The non-dimensional rotor separation distance (separation/2R) for the $\mathrm{V}-22$ is 1.226 while for the JVX it is 1.210. The JVX fuselage is a crude representation and nacelle/wing fairings are absent. Compared with the flight article, the CFD model has approximate nacelle/wing fairings, simplified flaperon cove seals, and no protuberances (VGs, strakes, probes). In the CFD model, the flap has been faired into the wing to completely seal the gap. The wing flaperon angle is set to 67 degrees and compared with data at the same setting. For simplicity, the nacelle inlet and exhaust locations have been modeled as solid surfaces rather than flow-through. Both full-span and half-span with image plane time-dependent CFD simulations were run. The intent is to investigate the differences between half- and full-span configurations, as might be tested in a wind tunnel.

\section{Grid Generation and Flow Solver}

The grids and flow solver operation are almost identical to that used for the dynamic isolated baseline analysis. Differences are noted here. The blade grid system used in the isolated hover analysis was installed directly on the V-22 with a nacelle angle of 90 degrees. Rotor rotation on each wing is such that the blades pass from wing leading to trailing edge. The distance between the spinner and blade root is smaller than for the isolated configuration. The nacelle spinner rotates with the blades, sliding over a portion of the nacelle spinner collar.

For the installed configurations, all near-body grids including the nacelles and fuselage are modeled as viscous. Viscous grid generation for the fuselage components relaxed the normal grid spacing due to significantly lower flow velocities and reduced wall-bounded viscous effects. The outer boundary spacing of the fuselage near-body grids matches with the finest off-body grid spacing, which remained at $0.10 c_{\text {tip }}$. The finest level off-body grids enclosed not only the rotor plane as in the isolated analysis but also the complete airframe. Figure 13 shows a streamwise crosssectional cut through the half-span volume grid system. The full-span grid system is approximately symmetric. The total number of grid points in the full-span model is 47.6 million with $63 \%$ in the off-body grids. The half-span model contains 23.6 million points. The $4^{\text {th }}$-order spatial scheme is used throughout. Double fringing is used in the blade and off-body grids while the fuselage near-body grids

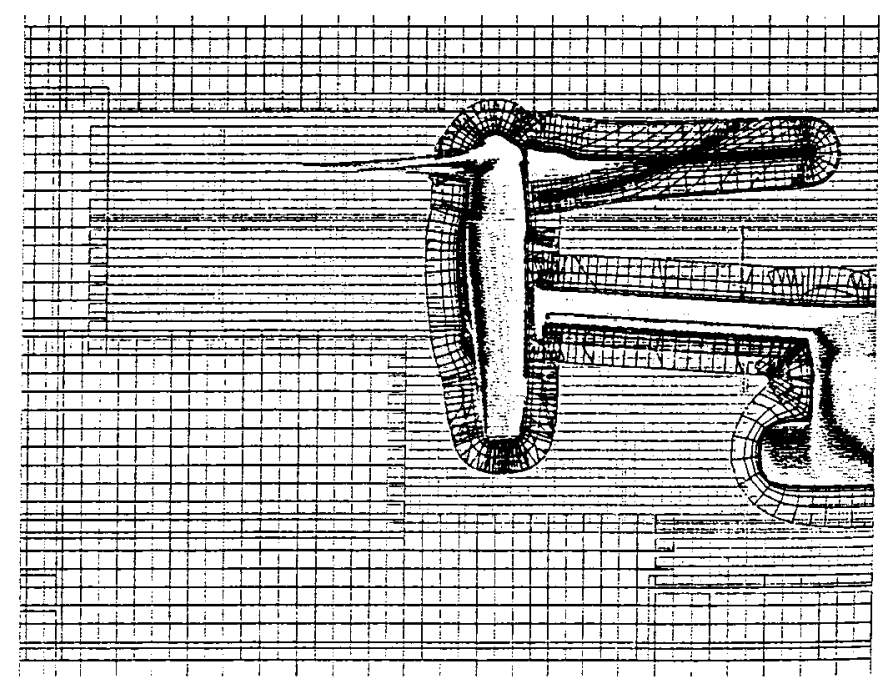

Figure 13. Slice through half-span V-22 volume grids (every third point). black - near-body, red - finest level off-body, blue - coarser level off-body 


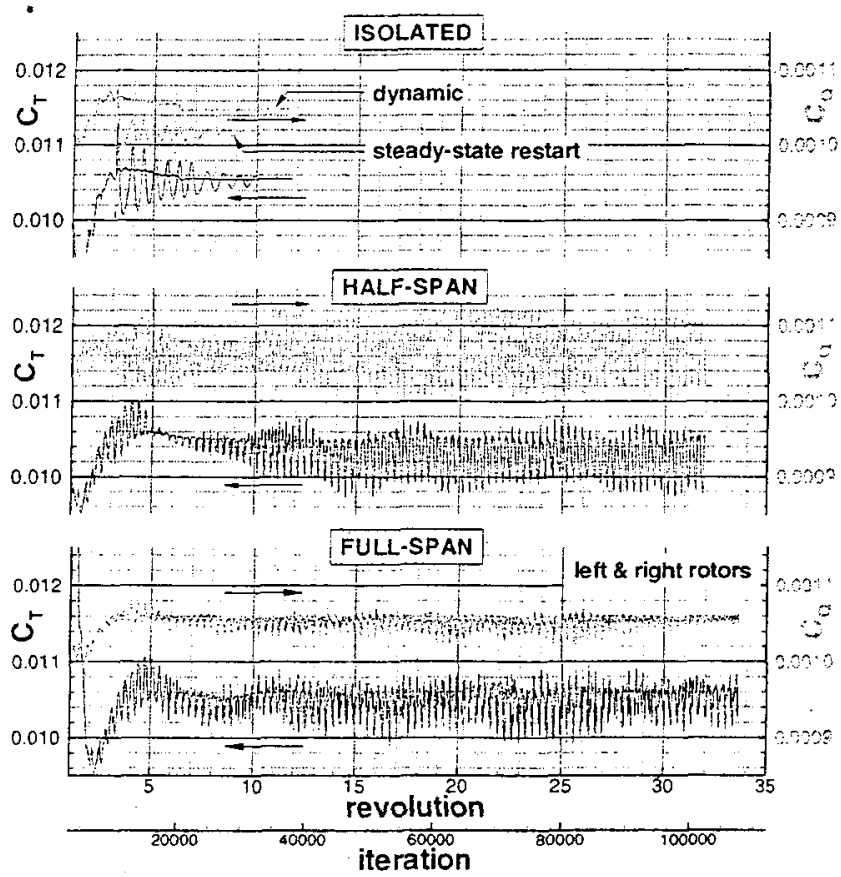

Figure 14. Isolated and installed TRAM rotor performance time histories at 10 degrees collective.

only have enough overlap for single fringing. Time advancement schemes and time step are unchanged. The installed simulations apply freestream characteristic conditions to the outer boundary. The half-span simulation has an inviscid plane of symmetry boundary condition at the centerline.

Both simulations were run for at least 31 rotor revolutions on 124 processors of an SGI Origin 2000. Each blade revolution requires 14.7 (full-span) and 8.6 (half-span) wallclock hours for 3200 iterations per revolution. Domain connectivity accounts for $20 \%$ of the wallclock time.

\section{INSTALLED HOVER RESULTS AND DISCUSSION}

The collective angle for the computed installed cases is 10 degrees with tip Mach number and Reynolds number unchanged at 0.625 and 2.1 million, respectively. Due to previously noted discrepancies in absolute performance between steady-state and dynamic isolated hover analyses, an isolated rotor dynamic simulation at 10 degrees collective was run for comparison with the installed configurations. Configuration increments from consistent, dynamic analyses should be unaffected.

\section{Rotor Performance}

Rotor performance for the isolated, half-span, and fullspan configurations are shown in Table 6 along with experimental data from the isolated TRAM DNW and JVX

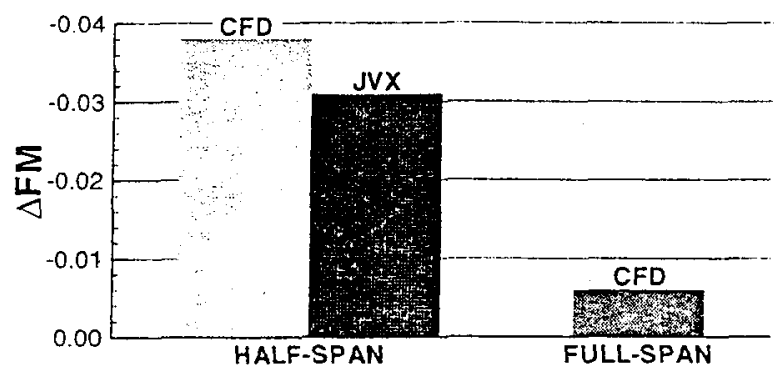

Figure 15 Installed vs. isolated rotor figure of merit increments.

Table 6. Performance comparisons of installed V-22 configurations. Calculations at 10 degrees collective.

\begin{tabular}{lcccc}
\hline \hline configuration & $\mathrm{C}_{\mathrm{T}} / \sigma$ & $\mathrm{C}_{\mathrm{O}} / \sigma$ & $\mathrm{FM}$ & $\Delta \mathrm{FM}^{\mathrm{a}}$ \\
\hline isolated - steady & .1002 & .00967 & .751 & \\
\hline isolated - dynamic & .1005 & .00999 & .730 & \\
\hline half-span & .0978 & .01021 & .688 & -.038 \\
\hline full-span & .1000 & .01002 & .724 & -.006 \\
\hline DNW TRAM & .1005 & .009505 & .768 & \\
\hline JVX isolated & .1005 & .009533 & .797 & \\
\hline JVX half-span & .1005 & .009921 & .766 & -.031 \\
\hline \hline
\end{tabular}

${ }^{2}$ installation $\triangle F M$ estimated at constant $C_{T}$

OARF tests. All FM increments due to rotor installation effects are estimated at constant thrust. Computational values for the installed configurations are averaged starting from the $20^{\text {th }}$ rotor revolution. Time histories of thrust and torque are shown in Figure 14 for steady-state and dynamic isolated, half-span, and full-span configurations. Timedependent solutions indicate a three per revolution oscillation with the largest oscillations for the half-span rotor.

The half-span analysis shows a 0.00028 loss in $C_{T}$ $(2.7 \%)$ at constant collective and a 0.038 reduction in $\mathrm{FM}$ compared with the isolated calculation. At the same $C_{T} / \sigma$, the IVX data for a half-span model without fuselage compared to an isolated rotor indicates a $0.031 \mathrm{FM}$ reduction. The full-span $C F D$ analysis shows almost no loss in thrust $(<0.5 \%)$ at constant collective and a much smaller performance penalty of 0.006 in FM compared with the isolated calculation. Hover performance increments due to rotor installation are summarized in Figure 15. Overall, the CFD calculations seem capable of correlating half-span installation increments with the available data. Comparison with accurate full-span increments should prove interesting. Absolute performance values are consistently underpredicted due to unresolved issues in the time-accurate simulations, although correlation improves somewhat when boundary layer transition corrections are applied. 


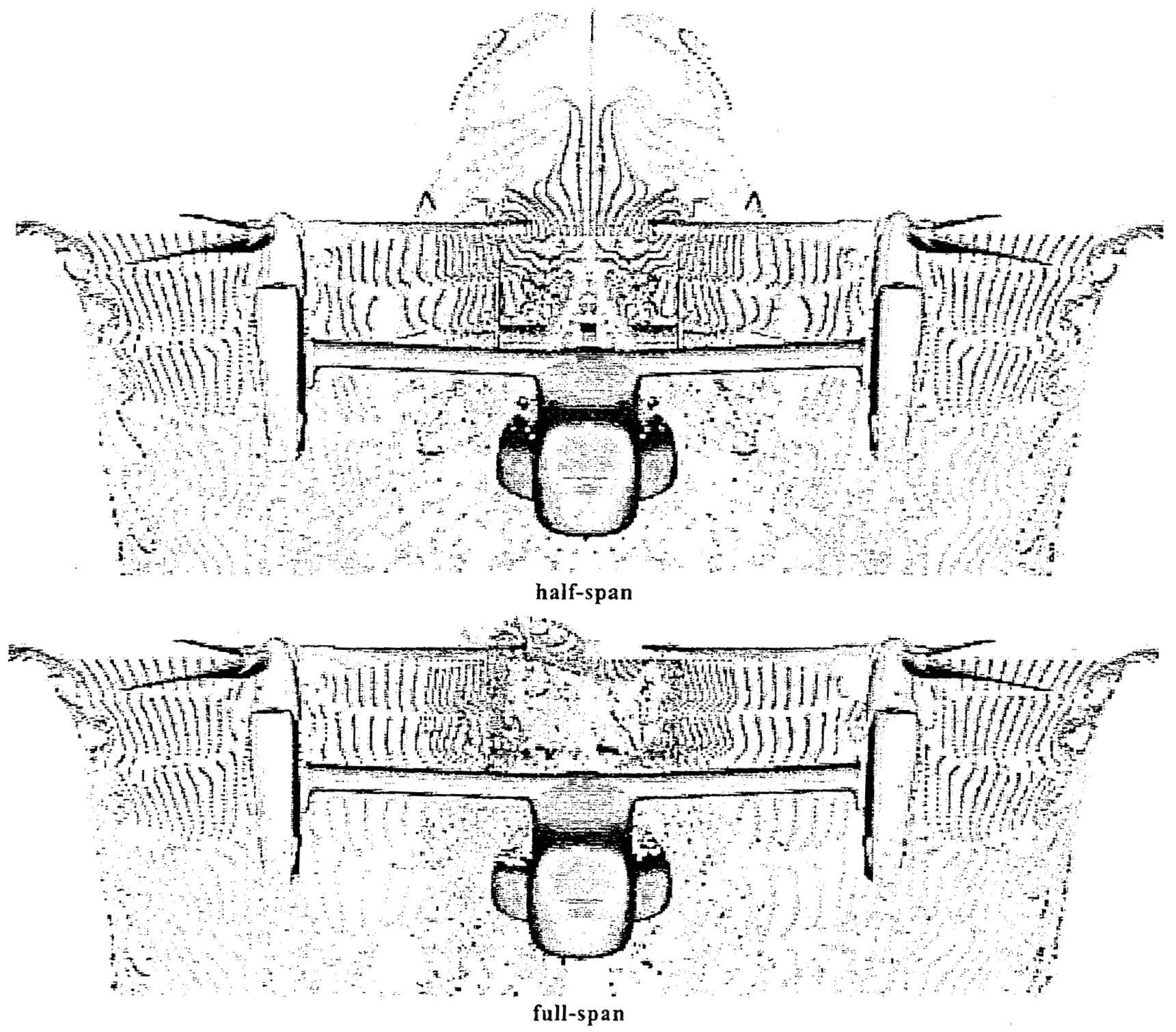

Figure 16. Time-dependent particle traces through V-22 simulations, colored by particle release time. blue - earliest, red - latest.

\section{Flowfield Details}

It is well known that there is an installation penalty due to a fountain of recirculating flow formed by the presence of the wing and second rotor, represented as either an image plane or full-span configuration. Some of the rotor downwash over the wing is deflected spanwise and inboard with the majority spilling over the wing leading and trailing edges. When the spanwise flow hits the centerline, it is deflected upward due to the image plane or spanwise flow from the opposite wing. The flow is then reingested into the rotor, reducing its performance as it passes over the wing.

Based on the time histories of the full-span analysis (Figure 14), the left and right rotor flowfields are initially symmetric and start becoming asymmetric after six rotor revolutions. Flow visualization indicates that the fountain is initially biased towards the left rotor but then switches to the right. The average calculated FM difference between the two rotors is only 0.002 . The asymmetry has been noted both experimentally [20] and computationally [7] with a frequency 1-2 orders of magnitude smaller than the rotor rotation frequency, which is just beyond the length of the current simulation.

The differences in half- and full-span fountain flows can be seen in the particle traces of Figure 16. Particles were released in a line connecting the rotor hubs and traced over three rotor revolutions. Due in part to the swirl component, the fountain is highly three-dimensional. The half-span calculation indicates a significantly larger fountain height and spanwise extent compared with the full-span calculation. The large spanwise extent is seen both above 
the rotor plane and in the disturbed flow behind and below the wing. Away from the fuselage, the organized wake of an isolated rotor is seen.

Representative instantaneous vorticity contours in a streamwise plane at the rotor centerline are shown in Figure 17. In the fountain region the tip vortices are initially convected towards the wing and then deflected upwards in the fountain. Several tip vortices can be seen in the recirculating flow. In the full-span case, the right rotor ingests the tip vortices from both rotors at this time step. For the half-span case, the larger size of the fountain and more disorganized region around the wing are easily seen. These features equate to a larger performance penalty compared with the full-span simulation. The blade wake can also been seen convecting down onto the wing. Overall, the characteristics of the two fountain flows match the experimental flow visualizations by Polak [20].

The recirculating flow and wing presence is evidenced in the blade loads in Figure 18. Away from the fuselage at azimuthal angles $(\Psi)$ of $30 \mathrm{deg}$ and $150 \mathrm{deg}(0 \mathrm{deg}$ along the streamwise axis and increasing in the direction of rotation), the loads are similar to the isolated analysis. There is no difference between the half- and full-span loads at these two locations. Over the fuselage ( $\Psi=270 \mathrm{deg})$, large reductions in outboard loading, especially for the halfspan case, indicate significant downwash from the recirculating region. This results in an overall thrust loss. Loadings inboard on the rotor blade while over the wing show a small increase due to the simulated ground effect.

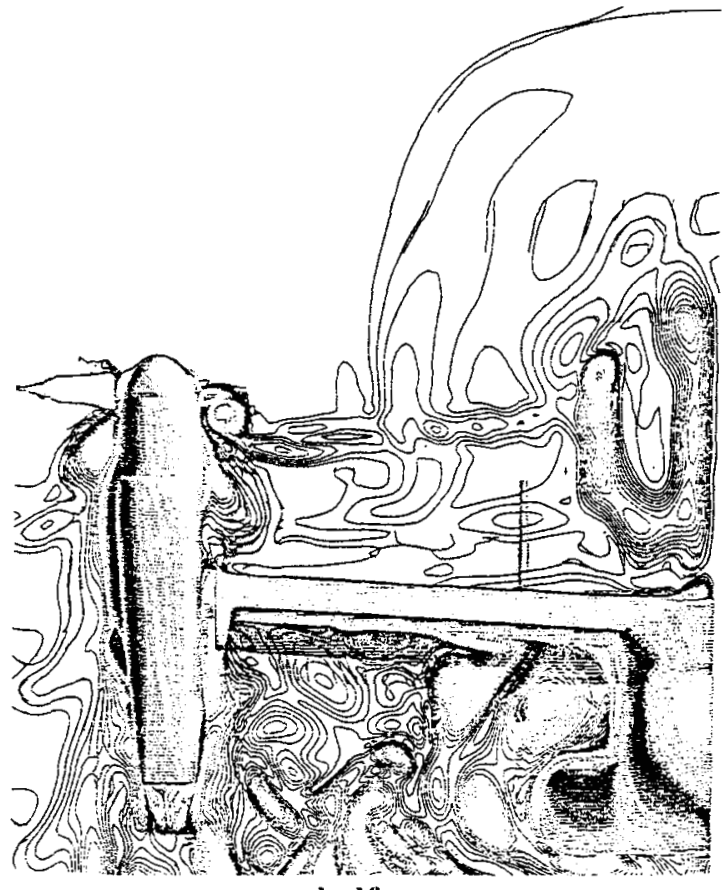

half-span
There is residual inboard loading remaining at $30 \mathrm{deg}$ azimuth. In the full-span simulation, this may counteract the reduced tip loadings over the wing enough to explain the small installed average thrust loss. It does not compensate for the even larger outboard thrust loss over the wing in the half-span case.

\section{Rotor-on-Wing Effects}

Airframe downloads for the installed configurations are shown in Table 7 along with experimental data from the JVX OARF test $\left(C_{T} / \sigma=0.100\right)$. The calculated average download includes all surfaces except the nacelle inlet and

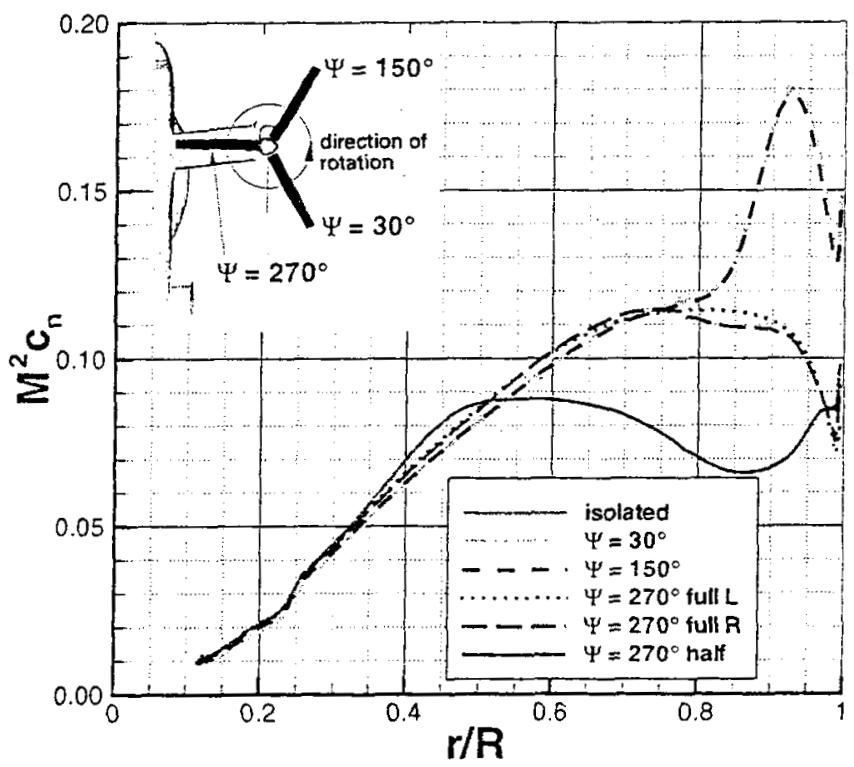

Figure 18. Isolated and installed TRAM rotor normal force distributions.

Figure 17. V-22 fountain vorticity contours. blue - low, red - high 
exhaust planes, which while modeled as solid are more realistically set to freestream pressure and accounted for in jet thrust. Values are averaged starting from the $20^{\text {th }}$ revolution. Time histories of aiframe forces are shown in Figure 19. Calculations indicate a rearward force that is trimmed out in flight with forward stick and/or nacelle tilt. Average side force for the full-span configuration is essentially zero.

The half-span CFD calculation shows increased download/thrust $(0.130)$ compared with the full-span calculation $(0.100)$. Download breakdown by component is also shown in Table 7, where the wing component extends only to the side of body $(\mathrm{r} / \mathrm{R}<1.01)$. The two CFD configurations carry the same download on the wings, so an additional load on the fuselage and sponsons accounts for the increased half-span total. The extra relief that is provided by the full span to reduce the fountain flow results in lower download, in addition to the previously discussed smaller rotor performance penalty. Taking into account configuration differences, the calculated wing component download (0.069) is in reasonable agreement with the JVX data which shows a wing download of 0.088 out of a total $0.106( \pm 0.01)$. The experimental wing download is calculated from integrated wing pressures only up to the side of body. The total includes an extrapolation to the image plane, accounting for the fuselage.

In Reference 21, the download breakdown between wing and fuselage on a semispan V-22 model with image plane was reported as $0.062(62 \%)$ and $0.038(38 \%)$, respectively, out of a total 0.100 . This data indicates that the half-span fuselage contribution from CFD is overpredicted. It is theorized that a viscous image plane might reduce the high velocities in the fountain flow. This would have the effect of reducing the fuselage download as well as increasing rotor performance. Nevertheless, overall download trends are reasonably well predicted by the CFD calculations given the scatter in available experimental data. The higher download on the half-span model is consistent with the physics of the computed fountain flows.

Figure 20 indicates the time-averaged pressure force in the download direction for the full-span V-22 calculation. A zero contour line separates regions of up- and download. The viscous contribution is negligible. The majority of the wing download occurs at the wing tips. A large upload region is seen at the flap hinge line and leading edge (not visible). Most of the fuselage download contribution is due to consistently negative pressures on the underside. Except in the fountain flow region, there is an upload on the topside of the fuselage. Flow spillage off the wing leading and trailing edges at the wing/fuselage fairing produces two download regions on the upper surface of the sponsons. Flow acceleration over the sponson sides creates an upload. The sponsons may be a worthwhile area of investigation for download reduction.
Table 7. V-22 download comparisons and breakdown by airframe component, $C_{\mathrm{T}} / \sigma=0.100$ ( - is upload).

\begin{tabular}{l|c|cc|cc}
\hline & JVX & \multicolumn{2}{|c|}{ half-span CFD } & \multicolumn{1}{c}{ full-span CFD } \\
\cline { 2 - 6 } component & DL/T & DL/T & $\%$ & \multicolumn{1}{c}{ DL/T } & $\%$ \\
\hline wing & .088 & .069 & 53 & .069 & 69 \\
\hline wing & .106 & & & & \\
\hline fuselage & & .034 & 26 & .015 & 15 \\
\hline sponsons & & .022 & 17 & .013 & 13 \\
\hline nacelles & & .004 & 3 & .005 & 5 \\
\hline tail & & .001 & 1 & .002 & -2 \\
\hline TOTAL & & .130 & & .100 & \\
\hline \hline
\end{tabular}

${ }^{2}$ integrated wing pressures and extrapolation to symmetry plane

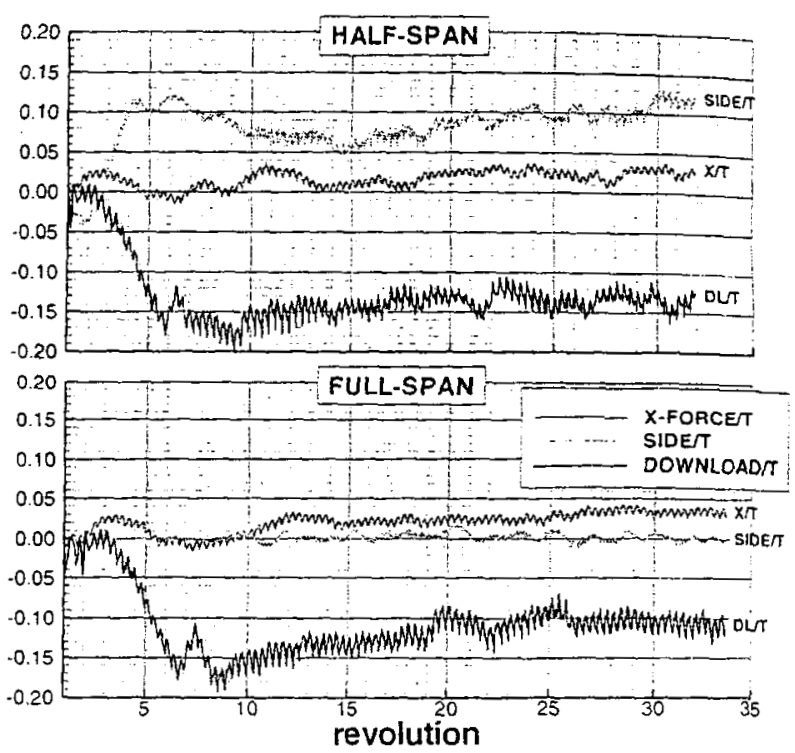

Figure 19. V-22 airframe force time histories.

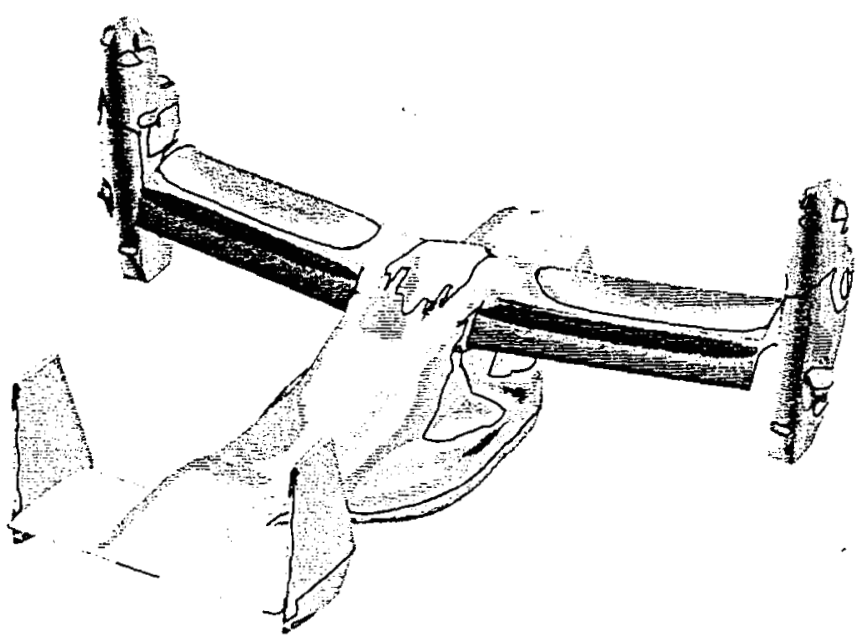

Figure 20. V-22 time-averaged airframe download pressure component. red - download, blue - upload, black line - zero contour 


\section{CONCLUSIONS}

Navier-Stokes CFD has been applied to isolated, halfspan, and full-span tiltrotor hover configurations, focusing on rotor performance, flowfield details, and airframe download. Guidelines have been given for grid generation and flow solver operation. The following conclusions are made from the data presented:

- Isolated tiltrotor performance across the thrust range is accurately predicted using a steady-state CFD formulation and taking into account boundary layer transition. Integrated forces are relatively insensitive to grid density, however, blade load distributions and wake geometry still show effects of numerical diffusion and cannot yet be validated.

- Flowfield and performance differences exist between steady-state and time-dependent, dynamic isolated hover solutions. The unresolved discrepancies have implications for the ability to calculate complex, installed tiltrotor configurations and comparison with isolated performance by steady-state calculations. Isolated rotor performance was consistently underpredicted by dynamic simulations, although incremental effects should not be affected.

- Calculations compared rotor performance and aiframe download between half- and full-span V-22 configurations. Differences can be traced to variations in the fountain flows. Download calculations show reasonable agreement with experimental measurements. The half-span rotor performance installation increment matches available test data.

- High-fidelity CFD methods provide a broad range of flowfield details, such as rotor sectional data, separated regions, and download distributions, that can be investigated to obtain improved performance from tiltrotor designs.

Future work is required and will focus on investigating the discrepancies discussed and expanding the CFD capabilities beyond performance prediction.

\section{ACKNOWLEDGEMENTS}

The computer resources of the NASA Advanced Supercomputing (NAS) Division, obtained through the Computing, Networking, and Information Systems (CNIS) Project, and the Department of Defense Major Shared Resource Centers (MSRC) are gratefully acknowledged.
1. Wake, B. E. and Baeder, F. D., "Evaluation of a Navier-Stokes Analysis Method for Hover Performance Prediction," Journal of the American Helicopter Society, Vol. 41, No. 1, January 1996, pp. 1-17.

2. Beaumier, P., Pahlke, K., and Celli, E., "NavìerStokes Prediction of Helicopter Rotor Performance in Hover Including Aero-Elastic Effects," American Helicopter Society $56^{\text {th }}$ Annual Forum, Virginia Beach, VA, May 2000.

3. Pomin, H. and Wagner, S., "Navier-Stokes Analysis of Helicopter Rotor Aerodynamics in Hover and Forward Flight," AIAA Paper 2001-0998, $39^{\text {th }}$ Aerospace Sciences Meeting and Exhibit, Reno, NV, January 2001.

4. Strawn, R. C. and Djomehri, M. J., "Computational Modeling of Hovering Rotor and Wake Aerodynamics," American Helicopter Society $57^{\text {th }}$ Annual Forum, Washington, DC, May 2001.

5. Fejtek, I. and Roberts, L., "Navier-Stokes Computation of Wing/Rotor Interaction for a Tilt Rotor in Hover," AIAA Journal, Vol. 30, No. 11, November 1992, pp. 2595-2603.

6. Poling, D. R., Rosenstein, H., and Rajagopalan, G., "Use of a Navier-Stokes Code in Understanding Tiltrotor Flowfields in Hover," Journal of the American Helicopter Society, Vol. 43, No. 2, April 1998, pp. 103-109.

7. Rajagopalan, R. G., "A Procedure for Rotor Performance, Flowfield and Interference: A Perspective," AIAA Paper 2000-0116, 38 th Aerospace Sciences Meeting and Exhibit, Reno, NV, January 2000.

8. Meakin, R. L., "Unsteady Simulation of the Viscous Flow About a V-22 Rotor and Wing in Hover," AIAA Paper 95-3463, Atmospheric Flight Mechanics Conference, Baltimore, MD, August 1995.

9. Chan, W. M., Meakin, R. L., and Potsdam, M. A., "CHSSI Software for Geometrically Complex Unsteady Aerodynamic Applications," AIAA Paper 2001-0593, 39 ${ }^{\text {th }}$ Aerospace Sciences Meeting and Exhibit, Reno, NV, January 2001. 
10. Meakin, R. L., "Automatic Off-Body Grid Generation for Domains of Arbitrary Size," AlAA Paper 2001$2536,15^{\text {th }}$ AIAA Computational Fluid Dynamics Conference, Anaheirn, CA, June 2001.

11. Strawn, R. C. and Ahmad, J., "Computational Modeling of Hovering Rotors and Wakes," AIAA Paper 2000-0110, 38 ${ }^{\text {th }}$ Aerospace Sciences Meeting and Exhibit, Reno, NV, January 2000.

12. Meakin, R. L. and Wissink, A. M., "Unsteady Aerodynamic Simulation of Static and Moving Bodies Using Scalable Computers," AIAA Paper 99-3302, $14^{\text {th }}$ AIAA Computational Fluid Dynamics Conference, Norfolk, VA, July 1999.

13. Johnson, W., "Calculation of Tilt Rotor Aeroacoustic Model (TRAM DNW) Performance, Airloads, and Structural Loads," Proceedings of the American Helicopter Society Aeromechanics Specialists' Meeting, Atlanta, GA, November 2000.

14. Young, L. A., Booth Jr., E. R., Yamauchi, G. K., Botha, G., and Dawson, S., "Overview of the Testing of a Small-Scale Proprotor," American Helicopter Society $55^{\text {th }}$ Annual Forum, Montreal, Canada, May 1999.

15. Swanson, S. M., McCluer, M. S., Yamauchi, G. K., and Swanson, A. A., "Airloads Measurements from a 1/4-Scale Tiltrotor Wind Tunnel Test," $25^{\text {th }}$ European Rotorcraft Forum, Rome, Italy, September 1999.

16. Felker, F. F., Signor, D. B., Young, L. A., and Betzina, M. D., "Performance and Loads Data from a Hover Test of a 0.658 -Scale V-22 Rotor and Wing," NASA TM 89419, April 1987.

17. Vassberg, J. C., Buning, P. G., and Rumsey, C. L., "Drag Prediction for the DLR-F4 Wing/Body Using OVERFLOW and CFL3D on an Overset Mesh," AIAA Paper 2002-0840, 40 ${ }^{\text {th }}$ Aerospace Sciences Meeting and Exhibit, Reno, NV, January 2002.

18. Wadcock, A. J., Yamauchi, G. K., and Driver, D. M., "Skin Friction Measurements on a Hovering FullScale Tilt Rotor," Journal of the American Helicopter Society, Vol. 44, No. 4, October 1999, pp. 312-319.

19. Felker, F. F. and Light, J. S., "Aerodynamic Interactions Between a Rotor and Wing in Hover," American Helicopter Society $42^{\text {nd }}$ Annual Forum, Washington, DC, June 1986.
20. Polak, D. R., Rehm, W., George, A. R., "Effects of an Image Plane on the Tiltrotor Fountain Flow," Journal of the American Helicopter Society, April 2000, pp. 90-96.

21. Wood, T. J. and Peryea, M. A., "Reduction of Tiltrotor Download," American Helicopter Society $49^{\text {th }}$ Annual Forum, St. Louis, MO, May 1993. 